\title{
USO DE MACROFUNGOS EM REGIÃO DE CAATINGA NO NORDESTE DO BRASIL
}

\section{Santina Barbosa de Sousa ${ }^{1 *}$, José de Ribamar de Sousa Rocha ${ }^{1,2}$, Reinaldo Farias Paiva Lucena ${ }^{3}$, Roseli Farias Melo de Barros ${ }^{1,2}$}

\author{
${ }^{1}$ Discente do Programa de Pós-Graduação em Desenvolvimento e Meio Ambiente (PRODEMA), Universidade Federal do Piauí. Núcleo de Referência em \\ Ciências Ambientais do Trópico Ecotonal do Nordeste (TROPEN). \\ ${ }^{2}$ Departamento de Biologia, Centro de Ciências Naturais. Universidade Federal do Piauí, Teresina, Piauí. \\ ${ }^{3}$ Departamento de Sistemática e Ecologia, Centro de Ciências Exatas e da Natureza, Universidade Federal da Paraíba. \\ *Autor para correspondência: sbarbosadesousa@gmail.com
}

Recebido em 30 de junho de 2016. Aceito em 07 de março de 2017. Publicado em 29 de julho de 2017.

\begin{abstract}
Resumo - O uso dos macrofungos por populações tradicionais tem destaque nas categorias alimentícia, medicinal e místico religiosa, sendo útil nas práticas cotidianas com importância para a qualidade de vida humana de tradições milenares que utilizam esse recurso. Objetivou-se conhecer e discutir usos dos fungos conhecidos e utilizados na comunidade Novo Zabelê, São Raimundo Nonato/PI, Brasil, inserida na Caatinga, a fim de obter-se conhecimento dos recursos fúngicos desta região. As coletas seguiram metodologia usual e foram incorporadas ao acervo do Laboratório de Micologia da Universidade Federal do Piauí (UFPI). O levantamento dos dados etnomicológicos foi realizado por meio de entrevistas semiestruturadas, conversas informais e turnês-guiadas. Foram referidas e identificadas oito espécies de macrofungos, distribuídas em três famílias, Ganodermataceae, Coprinaceae e Agaricaceae. As espécies que apresentaram maior Valor de Uso (VU) foram do Complexo Ganoderma applanatum (Pers.) Pat e Leucocoprinus sp. O maior conhecimento de uso micológico está contido na faixa etária adulta e idosa. O uso dos macrofungos, em alguns casos, foi semelhante ao de outras regiões, como México e China. A partir dos dados foi possível estimar a importância do conhecimento micológico, bem como a interação dos pesquisados com os bens naturais. Sugere-se que sejam levados em consideração os conhecimentos etnomicológicos em projetos de conservação da biodoversidade do Parque Nacional Serra da Capivara, do qual a comunidade estudada é oriunda, para preservar e valorizar o patrimônio biológico e cultural.
\end{abstract}

Palavras-chave: Etnomicologla; Utilização de macrofungos; Comunidade Rural; Conservaçấo.

THE USE OF MACROFUNGI IN CAATINGA REgION AT THE NORTHEAST OF BRAZIL

Aвstract - Macrofungi are used by traditional people in the food, medical and mystic-religious categories, being useful on daily practices, with significant importance for the millenary traditional human life quality, that use this resource. The present study aimed to know and discuss the uses of fungi known and utilized in Novo Zabelê community, São Raimundo Nonato, State of Piaú, Brazil, inserted in the Caatinga, in order to discover the fungal resources of this region. The sampling followed the usual methodology and was incorporated into the archive of the Mycology Laboratory of the Federal University of Piauí (UFPI). Ethnomycological data was carried out through semi-structured interviews, informal conversations and guided tours. Eight species of macrofungi were identified, distributed in three families: Ganodermataceae, Coprinaceae and Agaricaceae. The species that presented higher Value of Use (VU) were from the Complex Ganoderma applanatum (Pers.) Pat and Leucocoprinus sp. Adults and elder lies hold the greatest knowledge of mycological use. The use of macrofungi, in some cases, was similar to other regions, such as Mexico and China. From the data, it was possible to estimate the importance of mycological knowledge, as well as the interaction of the researchedones 
with the natural assets. It is suggested that ethnomycological knowledge should be taken into consideration in the Serra da Capivara National Park biodiversity conservation projects, from which the studied community is native, to preserve and value the biological and cultural heritage.

Keywords: Ethnomycology; Use of Macrofungi; RuRal Community; Conservation.

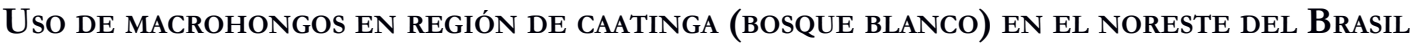

RESUMEN - El uso de macrohongos por poblaciones tradicionales tiene destaque en las categorías alimenticia, medicinal y místico-religiosa, siendo útil en las prácticas diarias con importancia para la calidad de vida humana de tradiciones milenarias que utilizan este recurso. Ese estudio tuvo como objetivo conocer y discutir usos de los hongos conocidos y usados en la comunidad de Novo Zabelê, São Raimundo Nonato / PI, Brasil, ubicada en la Caatinga, con el fin de obtener conocimiento de los recursos fúngicos de estaregión. Las recolecciones siguieron la metodología habitual y se incorporaron en el acervo del Laboratorio de Micología de la Universidad Federal de Piauí (UFPI). El levantamiento de los datos etnomicológicos se realizó a través de entrevistas semiestructuradas, charlas y visitas guiadas. Fueron reportadas e identificadas ocho especies de macrohongos, distribuidas en tres familias, Ganodermataceae, Coprinaceae y Agaricaceae. Las especiesque presentaron mayor Valor de Uso (VU) fuerondel Complejo Ganoderma applanatum (Pers.) Pat y Leucocoprinus sp. El mayor conocimiento de utilización micológica está contenido en los grupos de edad adulto y avanzado. El uso de macrohongos, en algunos casos, fue semejantes al de otras regiones, como México y China. A partir de los datos fue posible estimar la importancia del conocimiento micológico, así como la interacción de los investigadores con productos naturales. Se sugiere que deben tenerse en cuenta los conocimientos etnomicológicos en proyectos de conservación de la biodiversidad del Parque Nacional Serra da Capivara, del cual se deriva la comunidad estudiada, para conservar y valorizar el patrimonio biológico y cultural.

Palabras clave: Etnomicología; Uso de Macrohongos; Comunidad Rural; Conservación.

\section{INTRODUÇÃO}

O Brasil apresenta pequeno consumo de cogumelos, quando comparado a outros países, podendo ser atribuído tal fato a falta de tradição (Urben e Oliveira 1998). Isso implica a ausência de registros da utilização dos fungos pelos nativos que não foram registrados no período da colonização (Fidalgo 1985), sendo os primeiros registros descritos somente na década de 60, tendo como pioneiro Oswaldo Fidalgo (Fidalgo e Prance 1976).

O homem relaciona-se com os fungos desde as primeiras civilizações (Alexopoulos, Mimse Blackwell 1996). O uso desses organismos por populações tradicionais tem destaque nas categorias alimentícia, medicinal e mística, sendo útil nas práticas cotidianas com importância para qualidade de vida humana de tradições milenares na utilização nas diversas categorias de uso (Molitoris 1994), onde a aplicação tem relatos dos escritores da antiga Grécia, principalmente na aplicação médica e mística religiosa (Amazonas e Siqueira 2003).

Mundialmente, cerca de 20 espécies dominam o comércio de cogumelos comestíveis e dezenas de medicamentos à base de metabólicos fúngicos salvam ou prolongam a vida de milhares de pessoas, como o caso da penicilina que mudou a história da humanidade no século passado (Ishikawa et al. 2012).

O conhecimento das populações tradicionais é relatado no aspecto do uso de fungos comestíveis e da sua comercialização em mercados por populações rurais em regiões do México, onde para essas, os fungos representam importância para conservação da riqueza biológica, apresentando valor cultural e ecológico (Estrada-Martínez et al. 2009, Burrola-Aguilar 2012, Benítez-Badillo 2013, Mario et al. 2013, Bandala 2014). Encontram-se também 
relatos de uso tradicional de macrofungos na categoria gastronômica na região Colombiana, verificando saberes culturais semelhantes ao México (Peña-Cañón e Enao-Mejía 2014).

Os fungos comestíveis também são retratados em populações tradicionais amazônicas que apresentam saberes quanto à potencialização do cultivo de cogumelos, denotando informações de cunho cultural, biológico e ecológico acerca das espécies utilizadas, ressaltando que os primeiros estudos etnomicológicos brasileiros foram desenvolvidos com populações indígenas por consumiam fungos em sua dieta (Vargas-Isla et al. 2013; Vasco-Palacios et al. 2008; Fidalgo e Poroca 1986; Fidalgo e Prance 1976).

Por possuir propriedades nutricionais de grande valor, com utilização por povos orientais, os cogumelos vêm sendo introduzidos em hábitos alimentares usuais da população devido as suas substâncias bioativas com propriedades farmacológicas e baixo teor de gorduras (Orsine et al. 2012; Bett e Perondi 2011).

Os macrofungos possibilitam uso com potencial medicinal que são utilizados para cura das enfermidades na medicina popular (Gonzaléz 2013, Garibay-Orijel et al. 2010) e, que é demonstrado sua eficiência na medicina convencional no tratamento de pacientes com câncer, agregando valores com sua propriedade medicinal ou nutracêutica (Fortes et al. 2009, Abreu et al. 2015).

Uso de fungos em rituais religiosos como alucinógenos tem consumo tradicional associado às culturas há muito tempo, onde é considerado algo sobrenatural (Vargas-Isla et al. 2013). Fungos dessa natureza apresentam substâncias que atuam no sistema nervoso, alterando algumas percepções do tipo sensorial, estímulo externo e corporal (Wasson et al. 2008).

Os poucos registros existentes no Brasil, estão concentrados em populações indígenas. No Nordeste brasileiro não foi encontrado relatados de uso dos fungos. Nesse contexto, têm-se questionamentos se essa ocorrência tem origem na história de formação do território brasileiro e, ou ausência de pesquisadores interessados nessa área.

Na perspectiva de encontrar relatos sobre usos de fungos, pesquisou-se uma comunidade rural do sertão nordestino, objetivando levantar os usos dos fungos conhecidos e utilizados na comunidade, pressupondo que a população pesquisada reporta alguma forma de uso desses organismos que pode contribuir para o conhecimento da utilização dos recursos biológicos em região de Caatinga.

\section{Metodologia}

A pesquisa foi desenvolvida na Comunidade Nova Zabelê, município de São Raimundo Nonato, sul do Piauí (Figura 1). O município fica distante $530 \mathrm{~km}$ de Teresina, capital do Estado e, pertence a microrregião de São Raimundo Nonato, limitando-se ao norte com João Costa e Brejo do Piauí; ao sul com Fartura do Piauí; ao leste com Coronel José Dias e São Lourenço do Piauí e oeste com São Braz do Piauí e Bonfim do Piauí. O clima é tropical semiárido quente, com duração do período de estiagem de sete a oito meses e a vegetação é de caatinga arbórea e arbustiva (CEPRO 2010).

A formação da comunidade ocorreu de forma espontânea, localizando-se anteriormente no entorno dos paredões da Serra da Capivara, teve como atrativo inicial o extrativismo da maniçoba (Manihotsp), e distava 45 quilômetros da sede do município (Godoi 1999), constituído por grupo social secular, formado entre os anos de 1800 e 1900, cujo território teve que ser desocupado pelas famílias que lá viviam devido à implantação do Parque Nacional (PARNA) Serra da Capivara em 1989 (Matos 2012). Atualmente está situada em terras de 
assentamento rural denominado Nova Zabelê, criado pelo Programa de Reforma Agrária do governo brasileiro, em 1997. Embora a comunidade tenha se deslocado, o cenário ambiental atual permanece o mesmo, ou seja, continuam sobrevivendo na mesma fitofisionomia, com as mesmas condições ambientais.

Figura 1 - Mapa de localização da comunidade Novo Zabelê, São Raimundo Nonato/PI, Brasil.

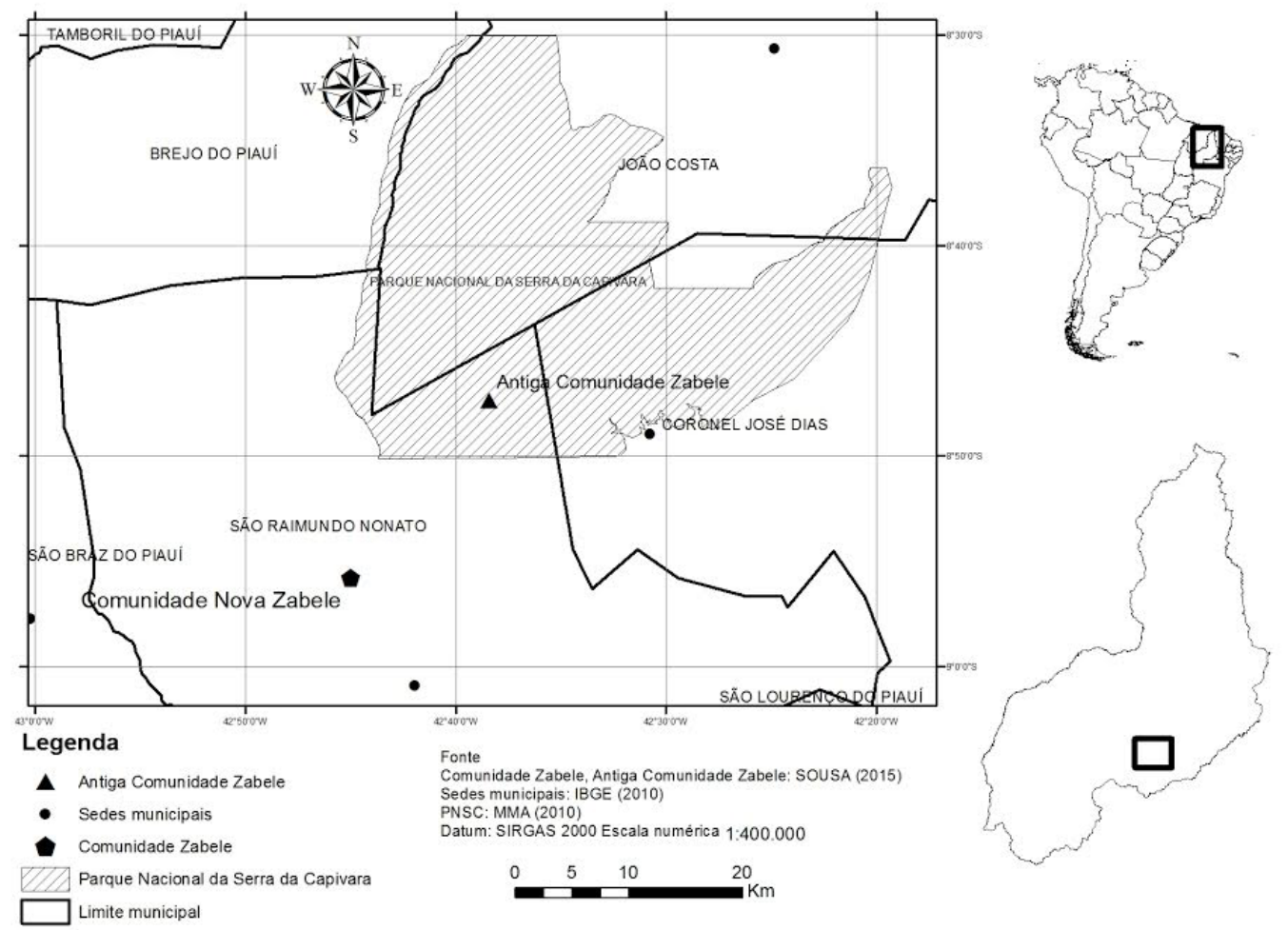

Optou-se por visitar todas as residências da comunidade Nova Zabelê com finalidade de restringir o universo amostral com os moradores provenientes da antiga comunidade Zabelê, em especial os que possuem notório saber sobre macrofungos, pressupondo que o conhecimento de uso é mantido por transmissão de geração em geração e, levando-se em consideração que os fatores históricos e culturais podem contribuir para esse conhecimento em região de Caatinga. Dessa forma, foram entrevistadas 35 famílias, incluindo pelo menos um mantenedor (pai ou mãe) e um filho maior de 18 anos, perfazendo um total de 56 moradores com faixa etária entre 19 a 90 anos, sendo 33 do gênero feminino e 23 do gênero masculino. Após a realização das entrevistas, foram visitadas todas as famílias que citaram uso das espécies de macrofungos, para obtenção de informações mais detalhadas.

A pesquisa foi aprovada pelo Comitê de Ética em Pesquisa (CEP) da Universidade Federal do Piauí (UFPI), com registro 36066214.4.0000.5214, atendendo ao que pressupõe a Resolução 466/2012 do Conselho Nacional de Saúde. A coleta de dados foi obtida com a utilização de entrevistas auxiliadas por formulário semiestruturado (Apolinário 2006) os quais concordaram em assinar o Termo de Consentimento Livre e Esclarecido (TCLE), no período de novembro de 2014 a abril de 2015.

As entrevistas semiestruturadas sobre conhecimento de uso de macrofungos seguiu-se com utilização projetiva de álbum seriado de fotografias das espécies locais (Medeiros et al. 2010), a fim de identificar os saberes etnomicológicos.

Aplicou-se a técnica da "Turnê-Guiada", preconizada por Bernard (1988), referida também por alguns autores como método informante de campo (Albuquerque et al. 2010), que consiste na realização de caminhadas 
no campo acompanhadas por moradores que possuíam maior conhecimento sobre o local e os macrofungos com referência de uso na região. Durante as turnês foram coletados exemplares do material biológico, conforme procedimentos preconizados por Vargas-Isla et al. (2014) e, Fidalgo e Bonine (1984). No Laboratório de Micologia da Universidade Federal do Piauí, identificou-se o material, por meio de observações macro e microscópicas, utilizando-se chaves analíticas contidas na literatura especializada, do qual se encontra incorporado ao acervo.

Os dados coletados foram organizados em tabelas e gráficos e analisados seguindo informações dos atores envolvidos na pesquisa, buscando comparar com bibliografias específicas. Assim, utilizou-se para cada espécie o cálculo do Valor de Uso mediante a fórmula ( $\left.\mathrm{VU}=\sum \mathrm{Ui} / \mathrm{n}\right)$ proposto inicialmente por Phillips \& Gentry $\left(1993^{a}\right)$ e, adaptado Rossato et al. (1999), onde: Ui= número de usos mencionados por cada informante, $\mathrm{n}=$ número total de informantes. $\mathrm{Na}$ análise houve distinção entre as citações de valor de uso atual (VU atual) e valor de uso potencial (VU potencial). Dessa forma, assume-se, por meio deste índice, a importância relativa do fungo pelo número de usos que apresenta.

Para o VU atual, considera os usos ainda realizados rotineiramente pelos informantes, e o VU potencial, considera os usos presentes na memória, mas, que não são mais utilizados por elas (Rossato et al. 1999; Lucena et al. 2009).

Para analisar o conhecimento por faixa etária, seguiu-se a delimitação utilizada pelo Instituto Brasileiro de Geografia e Estatística (IBGE, 2010): jovens (entre 18 e 24 anos), adultos (entre 25 e 59) e idosos (a partir dos $60)$.

\section{Resultado e Discussão}

Analisando os resultados obtidos, verificou-se que os macrofungos reconhecidos e utilizados pelos entrevistados da comunidade Novo Zabelê recebem citações, sobretudo, para cura de enfermidades (16\%) e uso combustível (2\%). Os que apenas reconhecem, mas, não citaram uso, corresponde 82\% (Figura 3).

Figura 2 - Citação de uso de macrofungos pelos informantes da comunidade Novo Zabelê, São Raimundo Nonato/PI.

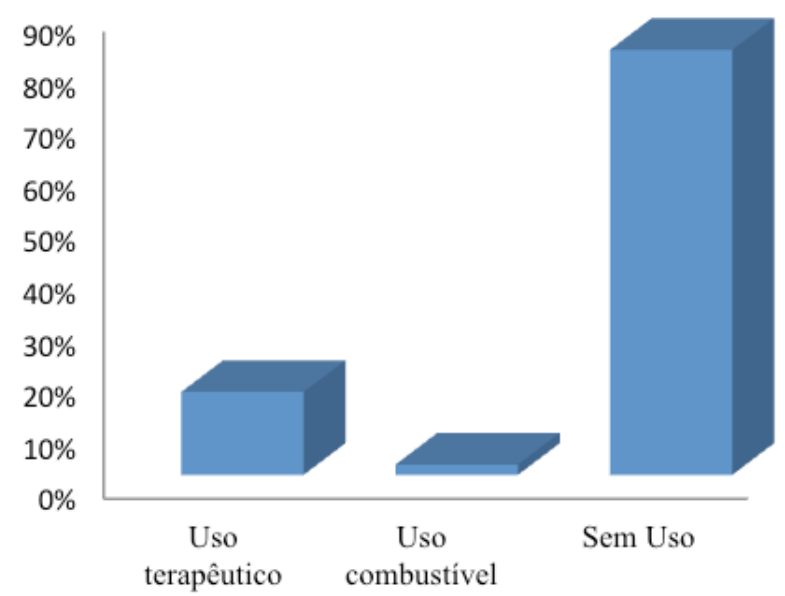

$\mathrm{Na}$ utilização dos fungos silvestres é recorrente o uso medicinal e, ocasionalmente o uso combustível. Foram identificadas sete espécies de macrofungos com citação de uso, listadas com sua respectiva forma de transmissão dos saberes (Tabela 1). As espécies do gênero Ganoderma foram agrupadas em dois complexos que 
reúnem espécies afins e de difícil determinação em nível específico, conforme relatado por Hapuarachchiet al. (2015), sendo 04 do Complexo Ganoderma applanatum (Pers.) Pat e 01 do Complexo Ganodema lucidum (W. Curt:Fr.) Karst.

Tabela 1. Citações de uso demacrofungos com forma de transmissão na comunidade Novo Zabelê - São Raimundo Nonato/PI. NC=Nome Científico; NL =Nome Local. NE= Número de Espécies. FT= Forma de Transmissão.

\begin{tabular}{|c|c|c|c|}
\hline NC & NL & NE & FT \\
\hline \multirow{6}{*}{$\begin{array}{l}\text { Ganodermataceae Com- } \\
\text { plexo Ganoderma applanatum } \\
\text { (Pers.) Pat }\end{array}$} & & & $\begin{array}{l}\text { "O meu tio fazia remédio para abortar" (E } 52 \\
\text { anos). }\end{array}$ \\
\hline & Orelha-de-pau & 04 & $\begin{array}{l}\text { "A orelha-de-pau serve para dor no pé da barri- } \\
\text { ga, quando está com problema no intestino" (E } \\
55 \text { anos). }\end{array}$ \\
\hline & & & $\begin{array}{l}\text { "Fazia chá para dor de barriga, quando as } \\
\text { crianças estavam com dor de barriga" (E } 78 \\
\text { anos). }\end{array}$ \\
\hline & & & $\begin{array}{l}\text { "Meu pai faz̧ia remédio da orelha-de-pau, } \\
\text { junto com pedaço da casca do angico" (E } 71 \\
\text { anos). }\end{array}$ \\
\hline & & & $\begin{array}{l}\text { "Meu avô fazia xarope de orelha-de-pau e a } \\
\text { casca do angico para gripe" (E } 49 \text { anos). }\end{array}$ \\
\hline & & & $\begin{array}{l}\text { "Ouvi falar na televisão que a orelha-de-pau } \\
\text { serve para transferi fogo" (E } 40 \text { anos) }\end{array}$ \\
\hline $\begin{array}{l}\text { Ganodermataceae Comple- } \\
\text { xo Ganoderma lucidum (W. } \\
\text { Curt:Fr.) Karst. }\end{array}$ & Orelha-de-porco & 01 & $\begin{array}{l}\text { "Meu avô colocava a orelha-de-porco em cima } \\
\text { de queimaduras para aliviara dor e em furo de } \\
\text { espinho para puxar o pus" (E } 55 \text { anos). }\end{array}$ \\
\hline $\begin{array}{l}\text { Agaricaceae } \\
\text { Leucocoprinus sp }\end{array}$ & Cogumel-do-sol & 01 & $\begin{array}{l}\text { "O cogumel-do-sol serve para o câncer. Eu usei } \\
\text { quando estava com câncer" (E } 50 \text { anos). }\end{array}$ \\
\hline & & & $\begin{array}{l}\text { "O cogumel-do-sol serve para remédio porque a } \\
\text { mãe tomou para o câncer" (E } 20 \text { anos). }\end{array}$ \\
\hline Agaricaceae & Bufa-do-cão & 01 & "No tempo em que remédio era dificil, eu usava \\
\hline Podaxis pistilares (L.:Pers) Fr & & & $\begin{array}{l}\text { o pó da bufa-do-cão que encontrava na estrada e } \\
\text { colocava na ferida braba" (E } 73 \text { anos). }\end{array}$ \\
\hline
\end{tabular}

Das famílias registradas, a Ganodermataceae foi mais bem representada nas citações, seguida da família Agaricaceae (Figura 4). Das espécies encontradas, todas são registradas pela primeira vez para o estado do Piauí.

Com base nas informações, o gênero Ganoderma (Ganodermataceae) aparece com maior citação de uso na categoria medicinal, sendo referido uso por membro da família, porém atualmente caiu em desuso. Observouse que tempos atrás o uso medicinal dos fungos ocorria com mais frequência devido os medicamentos convencionais serem de difícil aquisição, como observado na fala do informante: "No tempo que não tinha remédio fácil usava a bufa-do-cão para sarar ferida braba” (E 73 anos). O resultado é um indicativo que esse cenário mudou e 
que o uso tradicional diminuiu, podendo até deixar de existir em gerações futuras.

Figura 3 - Ilustração de Macrofungos com citação de uso na comunidade Novo Zabelê, São Raimundo Nonato/PI. A. Ganoderma sp1; B. Ganoderma sp2; C. Ganoderma colossum; D. Podaxis pistilares; E. Leucocoprinus sp.
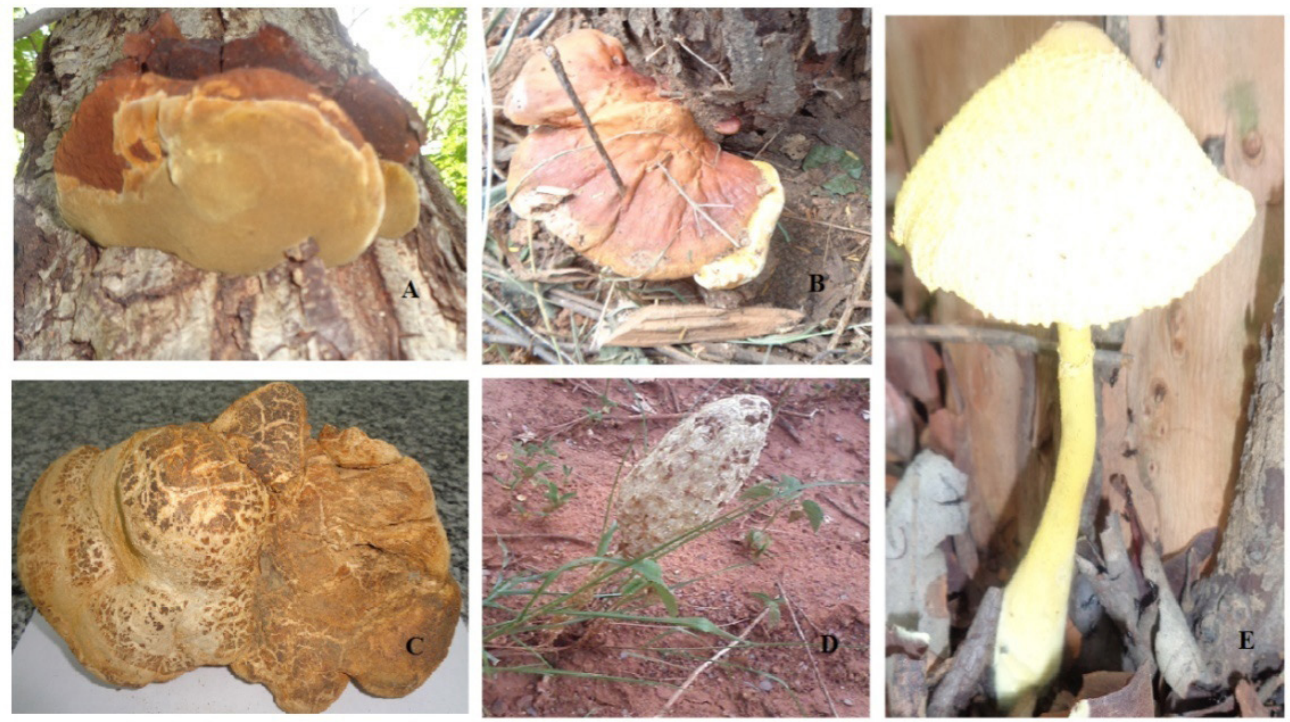

Os usos medicinais foram agrupados em cinco sistemas corporais, de acordo com a Organização Mundial da saúde - OMS (2000). O que representa maior Valor de Uso pertence ao Complexo Ganoderma applanatum (pers.) Pat com indicação para os sistemas digestivo, respiratório e reprodutor ( $V U=0,107)$ e, o gênero Agaricus $(\mathrm{VU}=0,053)$ citado com uso para o câncer (Tabela 2).

O índice de Valor de Uso descrito não indica que a espécie seja realmente utilizada, evidenciando apenas que tem ou teve um tipo de uso na comunidade. Nesse sentido, ocorreu mais citação de uso com espécies do gênero Ganoderma seguida do Leucocoprinus. Para o gênero Ganoderma o reconhecimento e indicação dos informantes foram para mais de um sistema corporal, como Aparelho Digestivo (infecção intestinal), Aparelho Respiratório (gripe, dor de cabeça e bronquite) e Aparelho Reprodutor (abortivo).

Informação de uso terapêutico para o gênero Ganoderma foi registrada em outras regiões como o continente asiático, onde tem sido utilizada amplamente por comunidades tradicionais, contribuindo para o conhecimento de uso, principalmente pelos povos da China, Japão, Ásia, Coreia (Miles e Chang 1997, Hapuarachchi et al. 2015; Singh et al. 2015), possuindo agente quimioterápico, com comprovação da ação de suas propriedades bioativas (Sliva 2003), sendo a espécie Ganoderma applanatum pode serusado como medicamento para várias doenças mortais (Acharya et al. 2005), utilizando como medicamento antigo de grande relevância para a era moderna (Russell e Paterson 2006), usada na região do México por populações tradicionais para tratamento de hemorragias nasais (Gonzaléz 2013), com princípio ativo comprovado, conferindo propriedade antibiótica, imuniestimulante, anticancerígena, hipoglicêmico, antihipertensivo e cardiotônico (Rogers 2011), ação antibacteriana (Moradali et al. 2006) e, antitumoral ( Sasaki et al. 1971, Acharya et al. 2005).

O uso tópico em ferimentos na pele, citado na pesquisa com espécie do Ganoderma lucidum (W. Cut: Fr) Karst., é reconhecida por suas propriedades farmacológicas, na medicina tradicional chinesa desde 4.000 anos e, estudada as inúmeras substâncias ativas como antialérgico, anti-tumorais e antiinflamatórios (Oliveira et al. 2003; Russell e Paterson 2006). Em estudos posteriores, concluiu-se que a natureza mais interessante deste tipo de fungo é a sua ação antitumoral (Lin e Zhang 2004). 
Tabela 2 - Utilização de espécies de macrofungos na comunidade Novo Zabelê - São Raimundo Nonato/PI, Brasil. NR= Número de Registro. CU= Categoria de Uso. CD= Categoria de Doenças. C= Citações. VUA= Valor de Uso Atual. VUP= Valor de Uso Potencial FP= Forma de preparação local.

\begin{tabular}{|c|c|}
\hline Espécie & $\mathrm{CU}$ \\
\hline
\end{tabular}

\begin{tabular}{|c|c|c|c|c|c|c|c|}
\hline 0019 & & & $\begin{array}{l}\text { Doenças do } \\
\text { Aparelho } \\
\text { digestivo e } \\
\text { Reprodutor }\end{array}$ & & & & $\begin{array}{l}\text { Colocar um pedaço da } \\
\text { orelha-de-pau na água } \\
\text { e fazer o chá. }\end{array}$ \\
\hline 0022 & $\begin{array}{l}\text { Complexo Ganoderma } \\
\text { applanatum (Pers.) Pat. }\end{array}$ & $\mathrm{M}$ & $\begin{array}{l}\text { Doenças do } \\
\text { Aparelho } \\
\text { respiratório }\end{array}$ & 6 & 0 & 0,107 & $\begin{array}{l}\text { Cozinhar a orelha- } \\
\text { de-pau completa } \\
\text { junto com a casca do } \\
\text { angico em } 1 \text { litro de } \\
\text { água, coar e voltar a } \\
\text { cozinhar com açúcar } \\
\text { para fazer o xarope. }\end{array}$ \\
\hline 0040 & Leucocoprinus sp & M & Anticancerígeno & 3 & 0 & 0,053 & $\begin{array}{l}\text { Colocar } 3 \text { pedaços } \\
\text { pequenos secos em } \\
\text { meio litro de água. } \\
\text { Depois de cozinhar } \\
\text { colocar mais meio } \\
\text { litro de água filtrada. } \\
\text { Colocar na geladeira e } \\
\text { beber durante o dia. }\end{array}$ \\
\hline 0027 & $\begin{array}{l}\text { Complexo Ganoderma } \\
\text { lucidum (W. Curt:Fr.) } \\
\text { Karst. }\end{array}$ & $\mathrm{M}$ & $\begin{array}{l}\text { Doença da Pele/ } \\
\text { Queimadura, } \\
\text { infecção. }\end{array}$ & 1 & 0 & 0,01 & $\begin{array}{l}\text { Uso tópico no } \\
\text { ferimento. Colocar } \\
\text { quando a orelha está } \\
\text { mole porque é fria. }\end{array}$ \\
\hline 0039 & $\begin{array}{l}\text { Podaxis pistilares } \\
\text { (L.:Pers) Fr }\end{array}$ & $\mathrm{M}$ & $\begin{array}{l}\text { Doença da Pele/ } \\
\text { ferimentos. }\end{array}$ & 1 & 0 & 0,017 & $\begin{array}{l}\text { Pegar o pó (esporos) } \\
\text { do fungo desidratado e } \\
\text { colocar no ferimento. }\end{array}$ \\
\hline
\end{tabular}

$\mathrm{Na}$ comunidade estudada a citação da categoria de doença anticancerígena foi relatada pelos informantes para o gênero Leucocoprinus, o qual foi referenciado por uma família em todas as faixa etária, que vivenciaram um caso de câncer de mama em um dos membros. Nas citações houve narração de cultivo e seleção do cogumelo para o uso medicinal, por meio de critério morfológico "Colocava bagaço da cana com estrume de cavalo e, aguava constantemente para nascer; o cogumel que serve é o todo de uma cor e mais duro que os outros, ele dá em estrume e bagaço de cana" (E 50 anos). O informante relata que depois de ingerir dosagem maior, passou mal e, a partir do ocorrido utilizou cogumelo desidratado industrializado. O gênero Leucocoprinus são venenosos e as escamas podem irritar os olhos (Puttzke 2014), isso justifica o motivo da informante sentir-se mal com ingestão do chá desse 
cogumelo. Espécies que pertencem a mesma família Agaricus sylvaticus (Schaeff) conhecido como cogumelo-dosol, possui ação terapêutica, que é utilizado no tratamento do câncer, por apresentar propriedades medicinais ou nutracêuticas, incrementando o seu valor agregado a medicina popular (Fortes et al. 2009, Abreu et al. 2015).

O Podaxis pistilaris (L.:Pers) Fr. foi reconhecido e identificado por um informante como fungo que cura ferimentos "brabo", nome conhecido na cultura popular nordestina para descrever um ferimento infeccionado. O uso foi citado, utilizando os esporos sobre a área afetada. Registro semelhante ocorreu em comunidades na região do México que utilizam espécie de fungos que se tornam puverulentos quando maduros como Lycoperdon (Pers.), Scleroderma (Pers.), Bovista (Pers.), Geastrum (Pers.), Pisolithus (Pers.), Astraeus (Pers.), Ustilago maydis (DC) Corda, com indicação de uso semelhante para infecção da pele (Gonzaléz, 2013).

As espécies de macrofungos com reconhecimento utilitário foram observadas pelos informantes no seu habitat natural e, o período em que estão disponíveis na natureza conforme citações "as orelhas-de-pau encontra todo tempo nos troncos dos paus" (E 55 anos); "a orelba-de-porco encontra no período de chuva em local de sombra" (E 55 anos); "a orelha encontra todo tempo na mata em qualquer pau e precisa da força do pau para crescer" (E 71 anos) e "a bufa-do-cão encontra em tempo de chuva na beira da estrada" (E 73 anos). As orelhas-de-pau foram as mais utilizadas, provavelmente em decorrência das condições climáticas locais serem favoráveis ao crescimento dessas espécies, aumentando sua disponibilidade na natureza ao longo do ano.

Além das citações de uso para categoria medicinal, houve um informante que atribuiu uso da espécie Ganoderma colosso, na categoria combustível. Em seu relato, informou que a orelha-de-pau que aparecia no cajueiro servia para transferir fogo de um lugar para o outro na roça e, que adquiriu a informação através da televisão.

A utilização para fins terapêutico dos macrofungos mostrou-se diferente entre os homens e mulheres, na comunidade pesquisada. Das citações de uso para fins medicinais, 78\% são mulheres, corroborando com registros em trabalhos etnobotânicos, onde há predomínio das mulheres no conhecimento sobre espécies medicinais (Lawrence et al. 2005, Lucena et al. 2012a, Lucena et al. 2012b). Os informantes maiores de 60 anos demonstraram maior conhecimento sobre os fungos utilizados, reconhecimento da forma e habitat, época que estão disponíveis e preparação, porém, entre a atual geração percebeu-se que houve redução do conhecimento intergeracional na maioria das citações de uso, evidenciando perda desse conhecimento nos jovens. Somente para o gênero Leucocoprinus foi citado o uso medicinal nas três gerações (avó, mãe e neto). Esses achados corroboram com resultados semelhantes em comunidades rurais colombiana, com o uso de fungos comestíveis, constando perda de conhecimentos na população mais jovem na tradição de consumo (Peña-Cañón e Enao-Mejía 2014).

Os relatos dos entrevistados apontam que a maioria dos informantes declara não usarem fungos, porém, não demonstraram aversão a eles, evidenciando que a ausência do uso pode estar mais ligada ao desconhecimento da utilização desses recursos do que serem considerados povos não micófilos.

\section{CoNSIDERAÇÕES FINAIS}

Os dados confirmam que os informantes têm conhecimento dos macrofungos, porém apenas 18\% reportaram algum tipo de uso, sobressaindo citações na categoria medicinal, demonstrando que a utilização terapêutica foi semelhante, em alguns casos, com populações tradicionais de outras regiões como México, China e Japão.

O achado da pesquisa suscita a ideia que os macrofungos incluem como um dos recursos na medicina 
popular em comunidade rural. As respostas de pessoas na faixa etária 60 à 70 anos, referindo uso por seus avós deixa evidente que o emprego das espécies como medicinais está caindo em desuso há pelo menos duas gerações, o que pode desaparecer. Nesse sentido, é importante que pesquisa no campo da Etnomicologia deva ser continuada em comunidades rurais brasileiras.

Levando em conta o fator histórico da área estudada no que diz respeito à ligação com o Parque Nacional Serra da Capivara, sugere-se que nas ações que envolvam a conservação da biodiversidade local seja levado em consideração o conhecimento tradicional dos macrofungos, a fim de incluírem na valorização do patrimônio natural e cultural.

\section{REFERÊNCIAS}

Abreu JAS, Rodovida AFS, Pamphile JA. 2015. Fungos de interesse: aplicações biotecnológicas. Review, vol. 21, n.1, pp.55-59.

Acharya K, Yonzone P, Rai M, Acharya R. (2005). Antioxidant and nitric oxide synthase activation properties of Ganoderma applanatum.Indian Journal of Experimental Biology, vol. 43, pp. 926-929.

Albuquerque UP, Lucena FPR, Alencar LN. 2010. Métodos e Técnicas para coleta de dados etnobiológicos. In: Albuquerque, U. P.; Lucena, R. F. P.; Cunha, L.V..F.C. 2010. (Orgs). Métodos e Técnicas na pesquisa Etnobiológica e Etnoecológica. Recife-PE: NUPEEA, 41-63 p.

Alexopoulos CJ, Mims CW \&Blackwell M. 1996. Introductory Mycology. New York: John Wiley \& Sons, Inc. $865 \mathrm{p}$.

Amazonas MALA, Siqueira P. 2003. Champignon do Brasil (Agaricus brasiliensis): Ciência, Saúde e Sabor. Empresa Brasileira de Pesquisa Agropecuária Centro Nacional de Pesquisa de Florestas Ministério da Agricultura, Pecuária e Abastecimento, ISSN 1517-526X.

Apolinário, F. 2006. Introdução à análise quantitativa de dados. In: Metodologia científica - filosofia e prática da pesquisa. São Paulo: Thomson Leaming.

Bandala V. M.; Leticia ML; Villegas R, Cabrera TG, Guitiérrz M. 2014. "Nanganana" "NANGAÑANA" (Tremelloscyphagelatinosasebacinaceae), hongo silvestre comestibledel bosque tropical decíduo em La depresión central de Chiapas, México. Acta Botanica Mexicana. vol.106, pp149-183.

Benítez-Badillo G, Alvarado-Castillo G, Nava-Tablada ME, Pérez-Vázquez A. 2013. Análisis del marco regulatorio en e laprovechamiento de los hongos silvestres comestibles en México. Revista Chapingo, vol. 19, n. 3, pp. 363-374.

Bett CF, Perondi MA. 2011. Análise do mercado de cogumelos comestíveis e medicinais: uma prospecção de alternativa de renda para a agricultura familiar na região sudoeste do Paraná. Synergis Muss Cyentifica UTFPR, Pato Branco, vol. 0 6, n. 1.

Burrola-Aguilar C, Montiel O, Garibay-Orijel R, Zizumbo-Villarreal L. 2012. Conocimiento tradicional y 
aprovechamiento de los hongos comestibles silvestres em la región de Amanalco, Estado de México. Revista mexicana de micologia, vol.35.

Campos CS, Andrade MCN. 2011. Aproveitamento de resíduos madeireiros para o cultivo do cogumelo comestível Lentinus strigosus de ocorrência na Amazônia. Acta Amazônica, vol. 41, n.1, pp.1-8.

CEPRO - Fundação Centro de Pesquisas. 2001. Perfil dos municípios, Teresina: Fundação CEPRO.

Estrada-Martinez E, Guzmán G, Cibrián TD, Ortega P R. 2009. Contribución al conocimiento etnomicológico de los hongos comestibles silvestres de mercados regionales y comunidades de La Sierra Nevada (México). Interciencia: Revista de Ciencia y Tecnología de América, vol.34, n.1, pp.25-33.

Fidalgo O. 1985. A história da Micologia Brasileira. In: II ENCONTRO NACIONAL DE MICOLOGIA, Recife-Pernambuco. Anais...Recife.

Fidalgo O, Prance GT. The Ethnomycology of the sanama Indians.1976. Mycologia, v. 68.

Fidalgo O, Poroca D J M. 1986. Etnomicologia brasileira. Boletim de Micologia, vol. 3, n.1, pp.9-19.

Fidalgo O, Bononi VL R. 1984. Técnicas de coleta, preservação e herborização de material botânico. Instituto de Botânica, São Paulo, 61 p.

Forte RC, Recôva VC, Melo AL, Novais MRCG. 2009. Qualidade de Vida de Pacientes com Câncer Colorretal em Uso de Suplementação Dietética com Fungos Agaricus sylvaticus após Seis Meses de Segmento: Ensaio Clínico Aleatorizado e Placebo-Controlado. Revista Brasileira Coloproct, vol. 27, n.2, pp.130-138.

Garibay-Origel R. 2012. Women care about local knowledge, experiences from ethnomycology. Journal of Ethnobiology and Ethnomedicine, vol.8, p.25.

Godoi PE. 1999. O trabalho da memória: cotidiano e história no sertão do Piauí. Campinas, SP: Unicamp, $55 \mathrm{p}$.

González JAB. 2013. Conocimiento tradicional de hongos medicinales em seis localidades deferentes del país. 158 f. Tese para obter título de Licenciado em Biologia. Faculdade de Ciências - Universidad Nacional Autótona do México. México.

Hapaurachchi KK, Wen TC, Deng CY, Kang JC, Hyde KD. 2015. Taxonomia Confusion in the Ganoderma lucidum Species Complex. Mycosphere, vol. 6, n.5, pp.542-559.

IBGE - INSTITUTO BRASILEIRO DE GEOGRAFIA ESTATÍSTICA. [Histórico dos municípios. [Acesso em junho de 2015]. Disponível em: http://www.cidades.ibge.gov.br/painel/historico.php?lang=\&dmu $\underline{\mathrm{n}}=221060$ \&search $=\mid$ sao-raimundo-nonato.

Ishikawa NK, Vargas-Isla R, Chaves R S, Cabral T S. 2012. Macrofungos da Amazônia: importância e potencialidades. Ciência \& Ambiente, vol.44, pp.129-139.

Lawrence A, Phillips OL, Reategui A, Lopez M, Rose S, Wood D e Farfan AJ. 2005. Local values for harvested 
forest plants in Madre de Dios, Peru: towards a more contextualized interpretation of quantitative ethnobotanical data. Biodiversity and Conservation, vol.14, pp.45-79.

Lin ZB, Zhang H. 2004. Anti-tumor and immunoregulatory activities of Ganoderma lucidum and its possible mechanisms. Acta Pharmacol, vol.25, n.11, pp.1387-1395.

Lucena CM, Costa GGS, Carvalho TKN, Medeiros Guerra NM, Quirino ZGM, Lucena RFP. 2012. Uso e conhecimento de cactáceas no município de São Mamede (Paraíba, Nordeste do Brasil). Revista de Biologia e Farmácia, vol. Especial, pp.121-134.

Lucena CM, Costa GM, Sousa RF, Carvalho TKN, Marreiros NA, Alves CAB, Pereira DD, Lucena RFP. 2012. Conhecimento local sobre cactáceas em comunidades rurais na mesorregião do sertão da Paraíba (Nordeste, Brasil). Biotemas, vol.25, n.3, pp.281-291.

Lucena RFP. Avaliando a eficiência de diferentes técnicas de coleta e análise de dados para a conservação da biodiversidade a partir do conhecimento local. Tese de doutorado. Universidade Federal Rural de Pernambuco. Recife. 190p.

Mario J R, Jesús PM, Juan JAS, Margarita TA. 2013. Hongos silvestres con potencial nutricional, medicinal y biotecnológico comercializados em Valles Centrales, Oaxaca. Revista Mexicana de Ciências Agrícola, vol.4, n.2, pp. 199-213.

Matos TCC. 2012. Imperativos político-normativos da política ambiental e da reforma agrária: impactos da comunidade Zabelê. In: XV ENCONTRO DE CIÊNCIAS SOCIAIS DO NORTE E NORDESTE e PRÉALAS BRASIL, 2012,Teresina-PI/Brasil. Anais...Teresina.

Miles PG, Chang ST. 1997. Mushroom biology: concise basics and current developments. Singapore: World Scientific, $194 \mathrm{p}$.

Medeiros PM, Almeida ALS, Lucena RFP, Souto FJB, Albuquerque UP. In: Albuquerque, U. P.; Lucena, R. F. P.; Cunha, L.V.F.C. 2010. O uso de estímulos visuais em Pesquisa etnobiológico (Orgs) Métodos e Técnicas na pesquisa Etnobiológica e Etnoecológica. Recife-PE: NUPEEA, p. 174.

Molitoris HP. 1994. Mushrooms in medicine. Folia Microbiologica, Prague, v. 39, n. 2, pp. 91-98.

Moradali MF, Mostafavi H, Hejaroude GA, Tehrani AS, Abbasi M, Ghods S. 2006. Investigation of Potential Antibacterial Properties of Methanol Extracts fron Fungus Ganoderma applanatum. Chenotherapy, vol.52, pp.241-244.

Oliveira P, Calado ML, Rosado J. Etnomicologia: uma breve introdução. 2003. In: Dias A S (Ed.). Etnobotânica. Perspectivas, história e utilizações. Universidade de Évora, Série Ciências da Natureza e do Ambiente, n. 4,pp. 39-44.

OMS - Organização Mundial de Saúde. 2000. Classificação estatística internacional de doenças e problemas 
relacionados à saúde. 10.ed. São Paulo: Editora da Universidade de São Paulo,1191p.

Orsine JVC, Brito L M, Novaes MRCG. 2012. Cogumelos comestíveis: uso, conservação, características nutricionais e farmacológicas. HCPA, vol.32, n.4, pp.452-460.

Peña-Cañón E R, Enao-Mejía L G. 2014; Conocimiento y uso tradicional de hongos silvestres de las comunidades campesinas associadas a bosques de roble (Quercushumboldtii) em la zona de influencia de la Laguna de Fúquene, Andes Nororientales. Etnobiologíavol.12, n.3.

Putzke, J. 2014. Cogumelos no sul do Brasil. Casa das letras, v. 1, 100p

Rogers R. 2011. The fungal pharmacy. The complete guide to medicinal mushroos and lichens of North America, North Atlantic.Books, Berkley, California.

Rossato SC, Leitão-Filho HF and Begossi A. 1999.Ethnobotany of caiçaras of the Atlantic Forest Coast (Brazil). Economic Botany, vol.53, n.4, pp.387- 95.

Russell R, Paterson M. 2006.Ganoderma - A therapeutic fungal biofactory. Phytochemistry, vol.67, pp.19852001.

Sasaki T, Arai Y, Ikekawa T, Shihara G, Fukuoka F. 1971. Antitumor Polysaccharides from Some Polyporaceae, Ganoderma applanatum (Pers.) Pat and Phellinuslinteus (Berk. et Curt.) Aoshima. Chem. Farm. Bull, vol.19, n.4, pp.821-826

SlivaD. 2003. Ganodermalucidum (Reishi) in câncer treatment. Integrative Cancer Therapies, vol.2, n.4, pp.35864.

Singh S, Harsh NSK, Gupta PK. 2015. Potencial role host tree species in determining the composition of polysaccharides of Ganodermalucidum (Fr.) Karst. (GTPSP).Current Research in Environmental \& Applied Mycology, vol.5, n.3,pp.196-201.

Urben AF, Oliveira C. (1998). Cogumelos comestíveis: utilização e fontes energéticas. In: REVISÃO ANUAL DE PATOLOGIA DE PLANTAS, 1998, Passo Fundo. Anais... Passo Fundo: [s.n.], v. 6, pp. 173-196.

Vargas-Isla R, Ishkawa N K, Py-Daniel V. 2013. Contribuição etnomicológico dos povos indígenos da Amazônia. Biota Amazônica, Macapá, v.3, n.1.

Vargas-Isla R, Cabral T S, Ishikawa N K. 2014. Instruções de coleta de macrofungosAgaricales e gateroides. Editora INPA, Manaus,30 p.

Vasco-Palacios A M, Suaza S C, Castaño-Betancur M, Franco-MolanoA E. 2008. Conhecimento etnoecológico de fungos entre os indígenas Uitoto, Muinane e Andoke da Amazônia Colombiana. ActaAmazônica, vol. 38, n., pp.17-30.

Wasson R G, Hofmann A, Ruck C A. 2008. The road to Elêusis - unveiling the secrets of the Mysteries. Berkeley(CA): North Atlantic Books. 